\title{
Use of the EKF for State Dependent Drift Estimation in Weakly Nonlinear Sensors
}

\author{
Arsenia Chorti*, Dimosthenis Karatzas, Neil White, Chris Harris
}

\begin{abstract}
A number of mechanisms are responsible for the generation of reversible or irreversible drift in the response of a sensor. In this letter, we discuss three approaches for the identification of reversible state dependent drift in sensors through the use of the Extended Kalman Filter. We compare their performance by simulation and demonstrate their validity by estimating the drift of an accelerometer, modeled as a weakly nonlinear system.
\end{abstract}

A. Chorti, D. Karatzas, N. White and C. Harris are with the Electronic Systems Design Group, Department of Electronics and Computer Science, University of Southampton, SO17 1BJ, UK (e-mails: ac2@ecs.soton.ac.uk, dk3@ecs.soton.ac.uk, nmw@ecs.soton.ac.uk, cjh@ecs.soton.ac.uk.)

Corresponding author: A. Chorti, ac2@ecs.soton.ac.uk 


\section{Use of the EKF for State Dependent Drift Estimation in Weakly Nonlinear Sensors}

\section{INTRODUCTION}

Various uncertain influences may generate drift in a sensor response, which, depending on the application, may constitute a significant source of unreliability. To mitigate these effects, an integral part of intelligent sensing is a drift compensation module [1]. A generic and universal approach for drift estimation cannot, however, be proposed due to the essentially different underlying mechanisms responsible for its generation. A variety of approaches have been discussed in the literature, including the use of classifiers based on Gaussian Mixture Models [2] and selforganizing maps [3], [4], as well as a hypothesis based use of the Principal Component Analysis decomposition [5].

In this letter, we attempt a classification for the different types of drift, which we believe is a prerequisite for any further analysis. In the case of state dependent reversible drift we argue that the Extended Kalman Filter (EKF) constitutes a simple and efficient baseline approach for drift estimation in weakly stationary sensors. We discuss three possible implementations of EKF-based drift estimators in the case of weakly nonlinear sensors and compare their performance in simulation. Finally our approach is validated by estimating the drift in real accelerometer output data.

\section{Algorithmic Drift Estimation}

We propose the categorization of drift in sensor systems into the following classes:

1) Reversible, state dependent drift.

2) Reversible, state independent drift.

3) Irreversible, state dependent drift.

4) Irreversible, state independent drift.

Drift in the latter category is due to the long-term mean of non-stationary intrinsic noise sources (such as $1 / f$ noise in electronic sensors) and in general it is the most difficult to identify and compensate.

Drift due to irreversible state dependent phenomena is the result of notable sensor non-stationarity (dynamic behavior) in the time scale of interest. Such drift can severely compromise the performance of a sensor system, such as an electronic nose. Common practice is to model the sensor dynamic behavior (e.g. expressed by an exponential decay model) and develop a coupled drift model [3]. Such drift generally follows a specific trend and an approach for its compensation is discussed by Vincent and Khargonekar in [6]. 
Alternatively, reversible state independent drift can generally be evaluated. A representative example of this class of drift is the gravitational offset (g-offset) in an accelerometer, generated from its relative to the ground angle. A commonly-encountered approach for the accelerometer calibration is through the use of a pair of accelerometers at known relative positions.

Reversible, state dependent drift arises from non-idealities in the sensor, the most common case being presented by nonlinearities. In the case where a weak-stationarity requirement is fulfilled, it is possible to develop approximate state space models of such sensors using the EKF. Hence, a tangible estimation of the mean drift/offset in the sensor output is possible. Let us assume that the sensor can be efficiently modeled by the state space equations:

$$
\begin{aligned}
\mathbf{x}_{k} & =\mathbf{A} \mathbf{x}_{k-1}+\mathbf{B} \mathbf{u}_{k-1}+\mathbf{w}_{k-1} \\
\mathbf{z}_{k} & =h\left(\mathbf{x}_{k}, \nu_{k}\right)
\end{aligned}
$$

with $\mathbf{x}, \mathbf{u}, \mathbf{w} \in \mathbb{R}^{n \times 1}, \mathbf{z}, \nu \in \mathbb{R}^{m \times 1} \mathbf{A}, \mathbf{B} \in \mathbb{R}^{n \times n}$ and $h(\cdot)$ being a mapping $\mathbf{R}^{m \times n} \rightarrow \mathbf{R}^{m}$. Then it is possible to approximate an offset in $\mathbf{z}$ as a function of the moments of $\mathbf{x}$ and $\mathbf{u}$ :

$$
E[\mathbf{z}]=g\left(E[\mathbf{x}], E\left[\mathbf{x}^{T} \mathbf{x}\right], E[\mathbf{u}], E\left[\mathbf{u}^{T} \mathbf{u}\right], \ldots\right)
$$

with $g: \mathbf{R}^{m \times n} \rightarrow \mathbf{R}^{m}$ and $E[\cdot]$ denoting statistical expectation.

We proceed by considering the specific case where the function $h(\cdot)$ is a low-order polynomial mapping, therefore the sensor is modeled as a weakly nonlinear system. Even order nonlinearities generate dc offsets that depend on the moments of the state $\mathbf{x}$ and of the control signal $\mathbf{u}$. In the case of Gaussian zero-mean states, we can directly evaluate the resulting dc terms by calculating the mean of the even order terms. For example the 2nd order nonlinearity will result in an output with a $\chi^{2}$ distribution and mean

$$
E\left[\mathbf{a}_{2}\left(\mathbf{x}^{T} \mathbf{x}+\mathbf{u}^{T} \mathbf{u}\right)\right]=\mathbf{a}_{\mathbf{2}}\left(\sigma_{x}^{2}+\sigma_{u}^{2}\right)
$$

while the 4th order nonlinearity will generate a dc offset of

$$
E\left[\mathbf{a}_{\mathbf{4}}\left(\mathbf{x}^{T} \mathbf{x}^{T} \mathbf{x} \mathbf{x}+\mathbf{u}^{T} \mathbf{u}^{T} \mathbf{u u}\right)\right]=3 \mathbf{a}_{\mathbf{4}}\left(\sigma_{x}^{4}+\sigma_{u}^{4}\right)
$$

with $\sigma_{x}^{2}$ and $\sigma_{u}^{2}$ being the variances of the processes $\mathbf{x}$ and $\mathbf{u}$ respectively.

As a specific case, we assume a weakly nonlinear system with Gaussian state variables, modeled by (1) and (2), with $\mathbf{x}=x \in \mathbb{R}, \mathbf{z}=z \in \mathbb{R}$ and

$$
h\left(x_{k}, \nu_{k}\right)=a_{0}+a_{1} x_{k}+a_{2} x_{k}^{2}+\nu_{k}
$$

Furthermore, we consider $A$ to be a constant linear gain, $B=0, w_{k}$ and $\nu_{k}$ to be zero-mean Gaussian noise processes with variances $Q$ and $R$ respectively and $a_{0}, a_{1}$ and $a_{2}$ to be constants in the polynomial representation of the system time characteristic. It follows that we only need to estimate the variance of the state space variable 
$x_{k}$. We present three possible implementations of the EKF algorithmic estimation of the drift due to the 2nd order nonlinearity.

In the first approach, labeled EKF1, the variance of the state variable $x$ is estimated as the sample mean of the variance of the a-posteriori state estimate $\hat{x}_{k}$, while the drift $d c_{k}$ at the $k$ th state is estimated as the instantenuous mean:

$$
\begin{aligned}
\underline{\mathrm{EKF} 1}: \sigma_{x}^{2} & \simeq \overline{\sigma_{\hat{x}_{k}}^{2}}=\frac{(k-1) \overline{\sigma_{\hat{x}_{k-1}}^{2}}+\hat{x}_{k}^{2}}{k} \\
d c_{k} & =a_{2} \overline{\sigma_{\hat{x}_{k}}^{2}}
\end{aligned}
$$

In the second approach, denoted as EKF2, the variance of the state variable is estimated as the instantenuous variance of the $k$ th a-posteriori estimate of the state variable while the drift is calculated as the sample mean up to the $k$ th state:

$$
\begin{aligned}
\underline{\mathrm{EKF} 2}: \sigma_{x}^{2} & =\sigma_{\hat{x}_{k}}^{2}=\hat{x}_{k}^{2} \\
\overline{d c_{k}} & =\frac{(k-1) \overline{d c_{k-1}}+a_{2} \sigma_{\hat{x}_{k}}^{2}}{k}
\end{aligned}
$$

In the third approach, both the variance and the drift are estimated from the sample mean values as below:

$$
\begin{aligned}
\underline{\text { EKF3 }: \sigma_{x}^{2}} & \simeq \overline{\sigma_{\hat{x}_{k}}^{2}}=\frac{(k-1) \overline{\sigma_{\hat{x}_{k-1}}^{2}}+\hat{x}_{k}^{2}}{k} \\
\overline{d c_{k}} & =\frac{(k-1) \overline{d c_{k-1}}+a_{2} \overline{\sigma_{\hat{x}_{k}}^{2}}}{k}
\end{aligned}
$$

If $x$ is a Gaussian random process, the algorithms EKF1 and EKF2 are equivalent and the estimated drift converges to the actual dc offset. The algorithm EKF3 represents a smoother approach and should be used only when it is desirable not to account for rapid dynamics in the process $x$. We will evaluate the performance of the three algorithms in the case of simulated Gaussian state variables and experimental sinusoidal state variables.

\section{Simulation Results with Gaussian StATE VARiables}

We simulate a weakly nonlinear system described through (1), (2) and (6) with the process $x$ being a zero-mean Gaussian random process, $(x \sim \mathrm{N}(0, Q))$, while we have set $a_{1}=1, a_{2}=0.01, Q=0.01$ and $R=0.0001$. In Fig. 1 the estimated drift through algorithms EKF1, EKF2 and EKF3 is compared to the empirical sample mean drift $\bar{z}=\frac{1}{n} \sum_{k=1}^{n} z_{k}$. The estimated drift through EKF1 and EKF2 are practically indistinguishable, with less than $0.05 \mathrm{~dB}$ difference and converge rapidly to the empirical mean drift after less than 250 samples. Whereas the drift estimated through algorithm EKF3 follows the trend of the empirical mean drift at a slower rate and has a residual offset.

In order to quantitatively evaluate the performance of the proposed algorithms, we illustrate in Fig. 2 and 3 respectively the absolute difference, averaged over 1000 runs, between the estimated drift through algorithms EKF1 


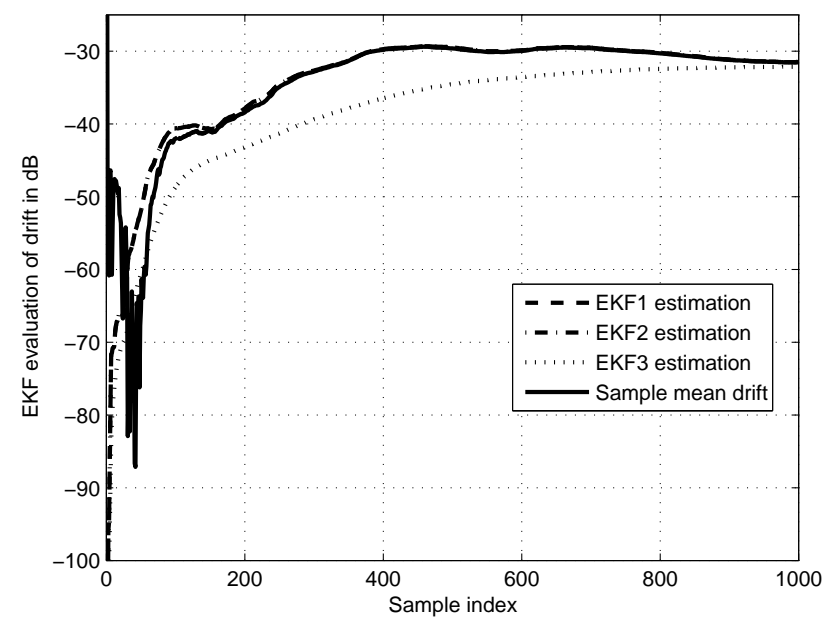

Fig. 1. Evaluated drift through algorithms EKF1, EKF2 and EKF3 and empirical drift evaluated as the sample mean drift. We have set $a_{1}=1, a_{1}=0.01, Q=0.01, R=0.0001$.

and EKF3 to the empirical drift as a function of the nonlinearity coefficient $a_{2}$ and the measurement noise variance $R$. The results obtained for EKF1 are almost identical to the results of EKF2 and as a result the latter are not included. In both cases better estimates are achieved for smaller values of $a_{2}$ and $R$, although EKF1 outperforms EKF3 in the case of Gaussian state variables and should be preferred.

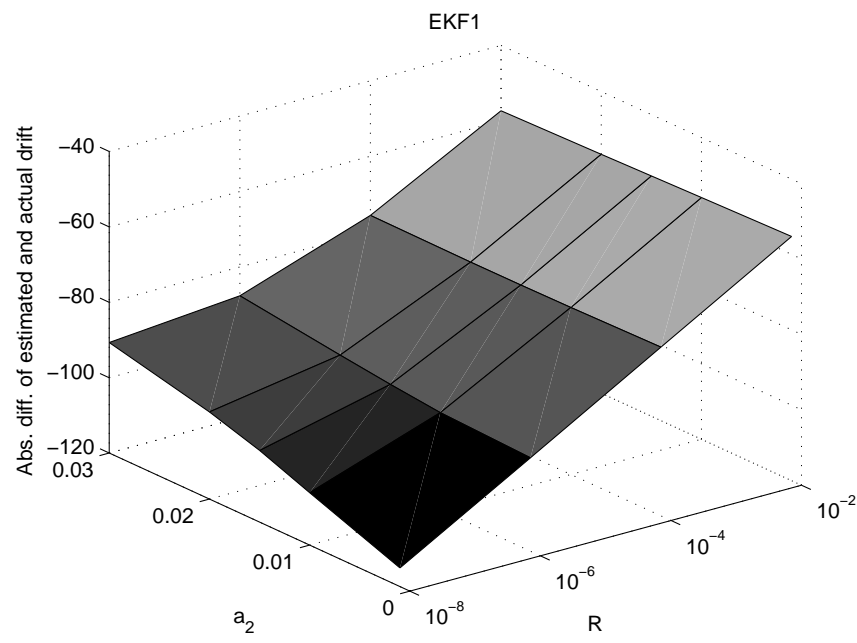

Fig. 2. Absolute difference between the estimated drift through algorithm EKF1 and the empirical drift evaluated as the sample mean as a function of the nonlinearity coefficient $a_{2}$ and the measurement noise variance $R$. We have set $a_{1}=1$ and $Q=0.01$.

\section{Measurement Results with Sinusoidal state Variable}

Based on spectral analyses of measurements from an Analog Devices ADXL203 dual-axis accelerometer, we derived a weakly nonlinear model for the accelerometer. It was driven by an acoustic vibrator of sinusoidal input 


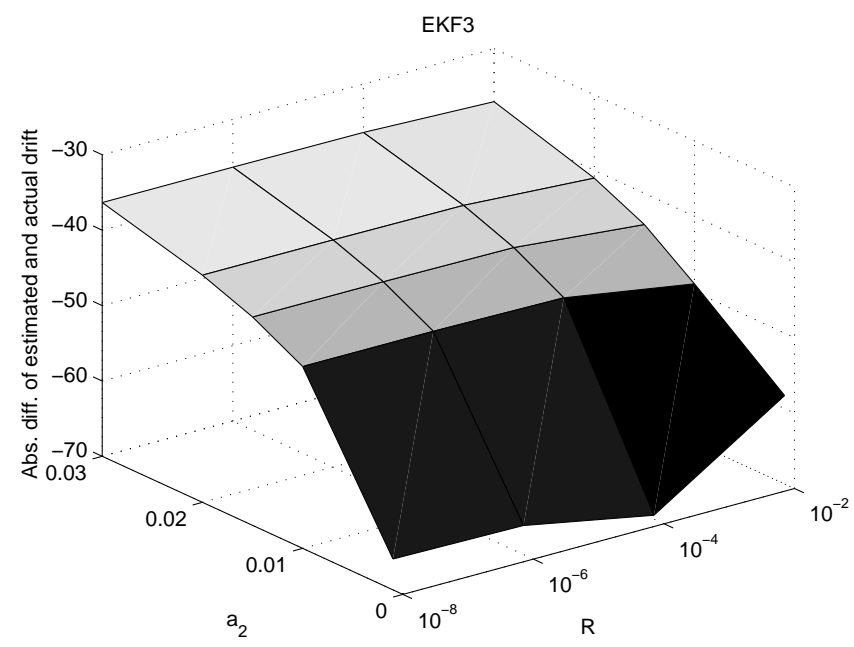

Fig. 3. Absolute difference between the estimated drift through algorithm EKF3 and the empirical drift evaluated as the sample mean as a function of the nonlinearity coefficient $a_{2}$ and the measurement noise variance $R$. We have set $a_{1}=1$ and $Q=0.01$.

at $\{100,125,150,175,200\} \mathrm{Hz}$ and we obtained $N=30$ sets of $n=60 \cdot 10^{3}$ measurements. We have used 25 of the sets to obtain a unique averaged 2 nd order model for the accelerometer, while using these parameters we algorithmically estimated the drift using EKF1 in the remaining 5 sets. In Fig. 4 we depict the estimated and empirical drift values for each of the remaining 5 sets for a driving frequency of $100 \mathrm{~Hz}$. The estimated drift follows the empirical drift in mean value, although the actual state variable in the specific experiment is sinusoidal and not Gaussian.

In Fig. 5 we have estimated the difference between the drift estimated using EKF1 and the mean empirical drift for all input frequencies, averaged over the five sets. The estimated drift is a very good estimate of the actual drift, with their difference being at least $5 \mathrm{~dB}$ lower than the actual drift for all input frequencies.

\section{CONCLusions}

In the case of a sensor that can be described by a state space model, it is possible to use an EKF-based algorithmic approach to estimate state-dependent drift. Specifically for an accelerometer, it has been shown that we can estimate the drift due to the 2 nd order nonlinearity through the use of a simple model. This approach can be extended to drift estimation resulting from axis cross-correlation in the case of a dual axis accelerometer. 

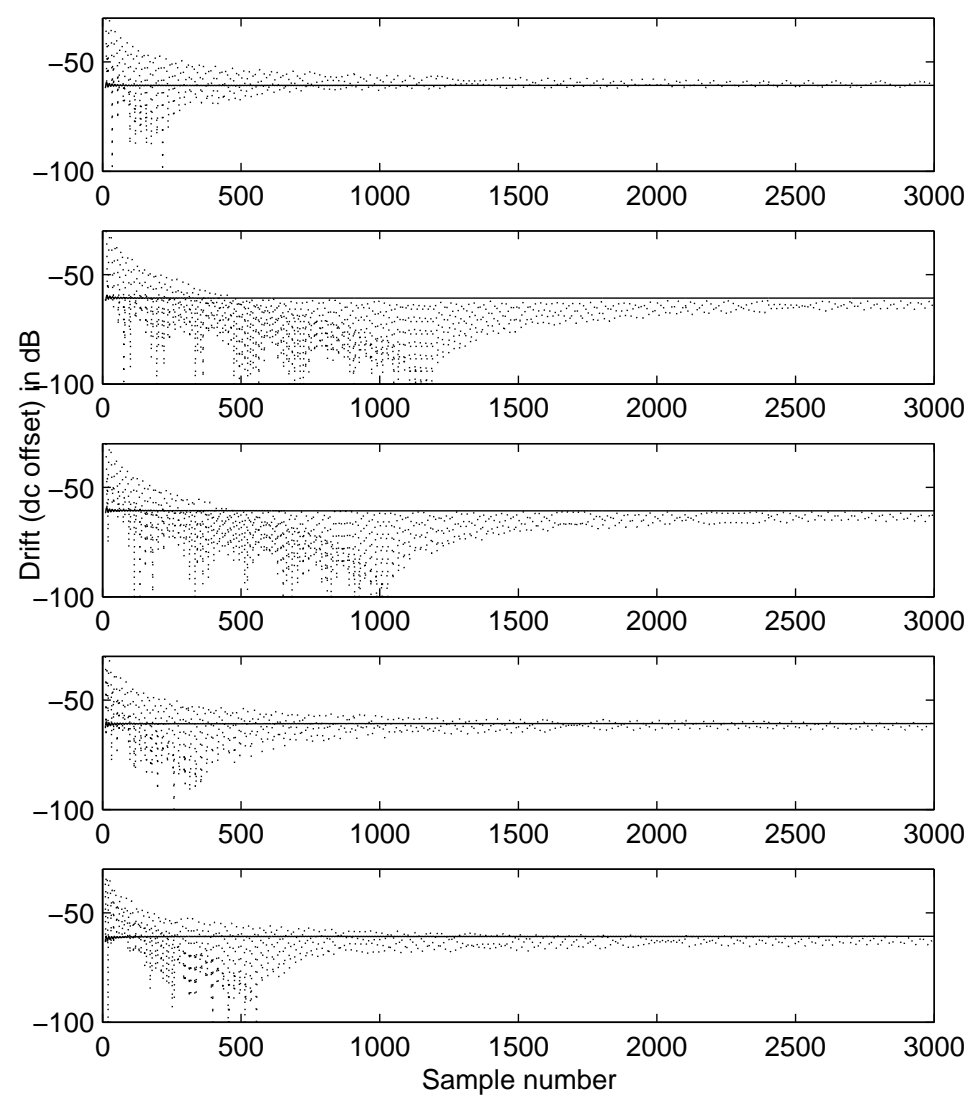

Fig. 4. Estimation of state dependent drift of an accelerometer using EKF1 driven by an acoustic vibrator at $100 \mathrm{~Hz}$. The solid lines represent estimations while the dot-lines are the empirical offsets.

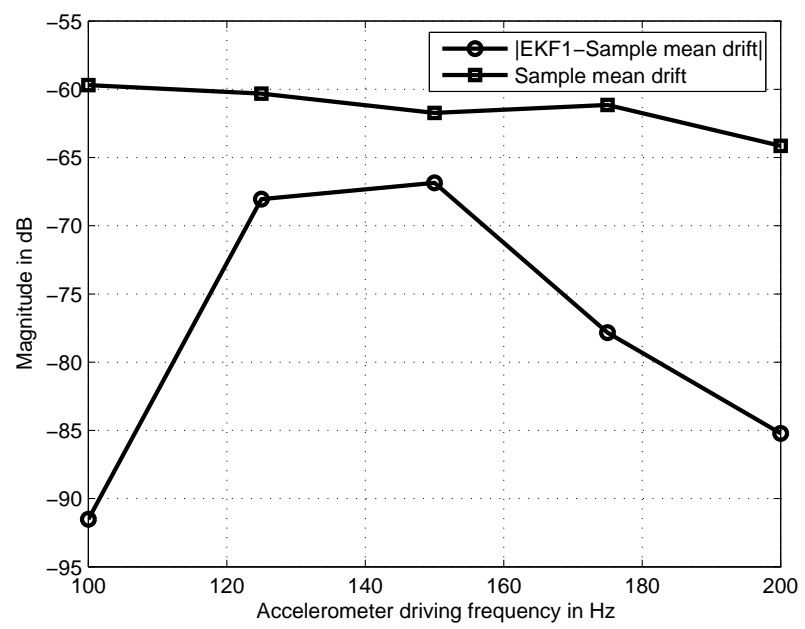

Fig. 5. Difference between the estimated drift using EKF1 and the empirical drift for an ADXL203 accelerometer for various driving frequencies. In the same graph the empirical drift is also depicted. 


\section{REFERENCES}

[1] M. Duta and M. Henry, "The fusion of redundant SEVA measurements," IEEE Transactions on Control Systems Technology, vol. 13, no. 2, pp. 173-184, Mar. 2005.

[2] S. Brahim-Belhouari, A. Bermak, and P. Chan, "Gas identification with microelectronic gas sensor in presence of drift using robust GMM," in Proc. of International Conference on Acoustics, Speech, and Signal Processing, ser. V, vol. 5. ICASP '04, IEEE, May 2004, pp. 833-836.

[3] S. Marco, A. Ortega, A. Padro, and J. Samitier, "Gas identification with tin oxide sensor array and self-organizing maps: adaptive correction of sensor drifts," IEEE Transactions on Instrumentation and Measurement, vol. 47, no. 1, pp. 316-321, Feb. 1998.

[4] M. Zuppa, C. Distante, P. Siciliano, and K. C. Persaud, "Drift counteraction with multiple self-organising maps for an electronic nose," Sensors and Actuators B, no. 98, pp. 305-317, Oct. 2003, Elsevier.

[5] T. Artursson, T. Eklov, I. Lundstrom, P. Martensson, M. Sjostrom, and M. Holmberg, "Drift correction for gas sensors using multivariate methods," J. of Chemometrics, no. 14, pp. 711-723, 2000.

[6] L. T. Vincent and P. P. Khargonekar, "A class of nonlinear filtering problems arising from drifting sensor gains," IEEE Transactions on Automatic Control, vol. 44, no. 3, pp. 509-520, Mar. 1999. 